\title{
$\$$ Research Square

\section{A Hypothesis about the Predominantly Gravitational Nature of Redshift in the Electromagnetic Spectra of Space Objects}

Stepan Tiguntsev ( $\nabla$ stiguncev@yandex.ru )

Irkutsk National Research Technical University

\section{Physical Sciences - Article}

Keywords: redshift, computational experiment, space gravitating object, photon, radiation source, receiver

Posted Date: December 30th, 2020

DOI: https://doi.org/10.21203/rs.3.rs-126341/v2

License: (c) (i) This work is licensed under a Creative Commons Attribution 4.0 International License.

Read Full License 


\title{
A HYPOTHESIS ABOUT THE PREDOMINANTLY GRAVITATIONAL NATURE OF REDSHIFT IN THE ELECTROMAGNETIC SPECTRA OF SPACE OBJECTS
}

\author{
Stepan G. Tiguntsev
}

\section{Irkutsk National Research Technical University}

83 Lermontov St., Irkutsk, 664074, Russia

stiguncev@yandex.ru

\begin{abstract}
The study theoretically substantiates the relationship between the redshift in the electromagnetic spectrum of space objects and their gravity and demonstrates it with computational experiments. Redshift, in this case, is a consequence of a decrease in the speed of the photons emitted from the surface of objects, which is caused by the gravity of these objects. The decline in the speed of photons due to the gravity of space gravitating object $(\mathrm{GO})$ is defined as $\Delta C=$ $C$ - $C$ ', where: $C^{\prime}$ is the photon speed changed by the time the receiver records it. Then, at a change in the photon speed between a stationary source and a receiver, the redshift factor is determined as $Z=\left(C-C^{\prime}\right) / C^{\prime}$. Computational experiments determined the gravitational redshift of the Earth, the Sun, a neutron star, and a quasar. Graph of the relationship between the redshift and the ratio of sizes to the mass of any space GOs was obtained. The findings indicate that the distance to space objects does not depend on the redshift of these objects.
\end{abstract}

Keywords: redshift, computational experiment, space gravitating object, photon, radiation source, and receiver.

\section{Introduction}

In the electromagnetic spectrum of space objects, redshift is assigned a significant role in creating a physical picture of the Universe. Redshift is observed in almost all space objects (stars, galaxies, quasars, and others). As a consequence of the Doppler effect, redshift indicates the movement of almost all space objects from an observer on the Earth [1]. Redshift is the main argument in favor of the theory of 
the expanding Universe and the theory of the Big Bang [2, 3]. Hubble's Law, which relies on the redshift concept, is employed to estimate the age of the Universe (in a very simplified and approximate way). Using redshift, researchers determined the higher rotation speed of galaxies, which gave rise to the concept of dark matter. The redshift concept also helped discover dark energy.

Redshift (a cosmological shift) is largely used as a magnitude characterizing the radial velocity $(V)$ of the space objects moving away in the Universe expanding after the Big Bang. In this case, the redshift is defined [4] by the expression:

$$
Z=\sqrt{\frac{C+V}{C-V}}-1
$$

and radial velocity is determined by the expression

$$
V=C^{*} \frac{(1+Z)^{2}-1}{(1+Z)^{2}+1}
$$

The value of the radial velocity is used to determine the distance to the object

$$
R=V / H
$$

where: $H$ is Hubble constant, $67.8(\mathrm{~km} / \mathrm{s}) /$ megaparsec $(1 \mathrm{megaparsec}=3260000$ light years).

Apart from the cosmological redshift, there is a concept of gravitational redshift, which is considered to be caused by gravitational time dilation, i.e., the energy loss by a photon transitioning to a region with a higher gravitational potential can be explained by the difference over time at the points of receiving and transmitting the signal [5].

Given that the gravitational shift of spectral lines does not have an unambiguous explanation accepted by all physicists, this research proposes a hypothesis - an explanation confirmed by computational experiments, which consider all the aspects of this phenomenon, including the relationship between the cosmological and gravitational redshift. 
The experiments involving the determination of a shift of the spectral lines tend to show the displacement in the frequency spectrum according to the expression $Z=\left(v_{o}-v^{\prime}\right) / v^{\prime}$ [6]. However, further, the researchers use a proportional relation between frequency and wavelength of a photon and take by default the indisputability of the constancy of the speed of light $C$ in the relation $\lambda=C / v(C$ is a speed of the photon (light), $v$ is a photon frequency). The shift factor, in this case, is defined by $Z=\left(\lambda^{\prime}-\lambda_{o}\right) / \lambda_{o}$, which for the shift toward the red end has the sign "+", and for the shift toward the blue end has the sign "-" (in the formulas, the subscript $(0)$ is for the source and superscript $\left({ }^{\prime}\right)$ is for the receiver).

However, the expression for the shift factor $Z=\left(\lambda^{\prime}-\lambda_{0}\right) / \lambda_{0}$ is incorrect because it is obtained considering the relations $\lambda_{o}=C / v_{o}$ and $\lambda^{\prime}=C / v^{\prime}$ from the expression $Z=\left(v_{o}-v^{\prime}\right) / v^{\prime}$ based on the indisputability of the constancy of the speed of light.

The hypothesis proposed in this paper is based on the assumption that the speed of light changes in the gravitational field. Moreover, if a photon moves away from a gravitating object (GO), i.e., an object with a gravitational field, the speed of the photon is decreased by the GO gravity, and if the photon moves towards the gravitating object, the photon speed increases.

To explain this assumption, let us consider how waves behave in a wave medium if this medium is in motion. To begin with, examine the case substantiating the Doppler effect [7]. Let the source of the waves be stationary, and the receiver be moving (no matter to or from the source). The source creates waves of a specific frequency and length, and during their movement, neither wavelength, nor frequency, nor the speed of the wave movement changes. However, the moving receiver detects waves with a frequency value different from the frequency value of the source. What will be the difference between the frequency at the receiver and the frequency at the source? The difference will be as large as large is the change in the total velocity of the receiver and the waves with respect to the velocity of waves $V$, i.e., as large as fast (or slow) will be the interaction between the receiver and the waves. Since $\lambda^{\prime}=\lambda_{o}, v^{\prime}=v_{o} *\left(V_{\text {wave }} \pm V_{r}\right) / V_{\text {wave }}$. 
Thus, $v_{o} / v^{\prime}=V_{\text {wave }} /\left(V_{\text {wave }} \pm V_{r}\right)$, i.e., the different frequency values at the receiver and the source are due to the additional velocity of waves because of the receiver's velocity $\Delta V=V_{r}$. In the case that the receiver approaches the source, the frequency value recorded at the receiver increases due to a rise in the total velocity $v^{\prime}=v_{o} *\left(V_{\text {wave }}+V_{r}\right) / V_{\text {wave. }}$ In this case, we have a blueshift, and shift factor $\mathrm{Z}$ will be

determined from the expression $v^{\prime}=v_{o} *\left(V_{\text {wave }}+V_{r}\right) / V_{\text {wave }}=v_{o} *\left(1+V_{r} / V_{\text {wave }}\right)=$ $v_{o} *(1+Z)$, i.e., $\mathrm{Z}=V_{r} / V_{\text {wave }}$

If the receiver moves away from the source, the frequency value recorded at the receiver declines due to a decrease in the total velocity $v^{\prime}=v_{o} *\left(V_{\text {wave }}-V_{r}\right) / V_{\text {wave }}$. In this case, we have a redshift, and shift factor $\mathrm{Z}$ will be determined from the expression $v^{\prime}=v_{o} *\left(V_{\text {wave }}-V_{r}\right) / V_{\text {wave }}=v_{o} *\left(1-V_{r} / V_{\text {wave }}\right)=v_{o} *(1-Z)$, i.e., $\mathrm{Z}= \pm V_{r} / V_{\text {wave }}$ $=\Delta V / V$.

\section{Waves in the wave medium in accelerated motion}

Let us consider the case where the receiver and the source are stationary with respect to one another, and there is a wave medium flow between them. The flow is accelerated, which provides an increment $\Delta V$ (or a decrease, depending on the direction of the waves with respect to the flow) of the wave velocity in the section between the source and the receiver. If the flow is uniform, there will be no velocity increment.

Such a phenomenon exists between a distant space object (a quasar, a galaxy, a star) and a receiver on the Earth.

Let us assume that each space gravitating object creates a very powerful flow of the light-carrying medium, which accelerates towards the gravitating object (gravity). In this flow, the light (photon) emitted from the surface of the gravitating object decelerates, and the speed of light (photon) goes down. The decline in the photon speed by the gravity of the space GO is defined as $\Delta C=C-C^{\prime}$, where $\mathrm{C}^{\prime}$ is a photon speed changed by the time of its recording at the receiver. Then, with a change in the photon speed between the stationary source and the receiver, the redshift is defined as 
$\mathrm{Z}=\Delta C /(C-\Delta C)=\left(C-C^{\prime}\right) / C^{\prime}$. In contrast to the case where the receiver moves towards the source, here, in the denominator, we use, which is logical, the speed of the photon it gained on the way from the source to the receiver, i.e., $C$ '. Then $C^{\prime}=C /(1+Z)$.

From the expressions $Z=v_{0} / v^{\prime}-1$ and $C^{\prime}=C /(1+Z)$ we obtain $v_{0} / v^{\prime}=C / C^{\prime}$. Given the difference between the photon speed $C$ and constant $C$, the expression for the photon frequency at the source has the form $v_{0}=C / \lambda_{0}$, and, accordingly, at the receiver $v^{\prime}=C^{\prime} / \lambda^{\prime}$. By substituting them in the expression $v_{0} / v^{\prime}=C / C^{\prime}$, we will obtain that the relationship between the wavelength at the source and the receiver will be defined by the expression $\lambda_{0}=\lambda$, i.e., the photon wavelength does not change when the photon moves in the gravitational field.

The obtained relation $\lambda_{0}=\lambda^{\prime}$ indicates that the expression $Z=\left(\lambda^{\prime}-\lambda_{0}\right) / \lambda_{0}$, which is used in physics to determine the shift factor through the values of photon wavelengths at the source and the receiver, is incorrect.

Thus, the wavelength and frequency of the photon at the receiver are determined by the expressions $\lambda^{\prime}=\lambda_{0}$ and $v^{\prime}=v_{0} *\left(C^{\prime} / C\right)$ if $C^{\prime}=C /(1+Z)$, i.e., the value of the photon frequency recorded at the receiver differs from the value of the photon frequency at the source. At the same time, if the photon speed declines (photon moves against the gravity forces), the photon frequency value recorded at the receiver is decreased by a factor of $\left(C^{\prime} / C\right)$. If the photon speed rises (the photon moves along the gravity force action), the value of the photon frequency recorded at the receiver is increased by a factor of $\left(C^{\prime} / C\right)$. In this case, the expression $\lambda^{\prime} * v^{\prime}=\lambda_{0} * v_{0} * C^{\prime} / C$ will hold true.

The physics of such a process can be explained as follows: the receiver captures a lower number of peaks of the waves traveling in a moving wave medium if the waves move against the medium movement (decelerate). Accordingly, the receiver records a larger number of peaks of the waves if they 
move in the direction of the medium movement (accelerate). In this case, the frequency of the photon relative to the moving medium does not change.

We observe an effect, which is physically similar to the Doppler effect but differs from it by that it exists between the wave source and receiver that are stationary with respect to one another.

\section{Frequency change in the electromagnetic spectra of space objects}

From the perspective of the revealed effect, we should explain why and how an observer on the Earth registers changes in frequency (wavelength) in the electromagnetic spectra of space objects.

Note that the frequency meters (devices designed to obtain electromagnetic spectra from space objects) are calibrated to measure frequency, provided that $v=C / \lambda$, where $C=299792458 \mathrm{~m} / \mathrm{s}$, and they, when adjusted, cannot use any other value of the speed of light because the speed of any photon emitted by any atom of the calibration source is $C=299792458 \mathrm{~m} / \mathrm{s}$.

The process is as follows. A signal of the reference frequency is sent to device $v_{1}$ and a mark on the device scale is made (exaggeratedly), a signal of a different frequency $v_{2}$ is sent, and another mark is made. Thus, the whole scale is marked.

Now, if a signal with frequency $v_{i}$ arrives at the device, it is undoubtedly measured subject to the speed of the signal equal to $\mathrm{C}$ (and no other speed is assumed at all).

What happens when a signal arrives at a speed other than the constant? The device registers the frequency $v^{\prime}=v_{0} * C^{\prime} / C$, other than the frequency of the source (because it cannot choose the necessary figure out of these three multiplied ones $v_{0}, C^{\prime}, C$, which is why it registers the final value), although, in actuality, the frequency of the signal with respect to the moving medium has not changed. If the 
device were calibrated at the speed of light equal to $C^{\prime}$, it would show the frequency $v_{0}$. We have already learned that the wavelength of the signal does not change, i.e., $\lambda^{\prime}=\lambda_{0}$.

However, astrophysical instruments are normally calibrated not in terms of frequency but in terms of wavelengths [8]. In this case, the wavelength is calculated by the expression $\lambda_{i}=C / v_{i}$ and the electromagnetic spectrum is plotted in terms of wavelengths, i.e. shows the change in wavelength. For example, with redshift, the recorded frequency decreases in comparison with the emitted one, and the device allegedly indicates an increase in the wavelength.

We can make sure that the frequency of radiation depends on the wave speed by a simple reduction of the speeds. It is sufficient to multiply the value of the frequency recorded by the device by the value $C / C$ and then we will see frequency $v_{0}$. In this case, $C / C^{\prime}=1+Z$.

Thus, the wavelength does not change when light moves from a distant space object. The changes occur only in the speed of light and in the recorded frequency when the speed of light changes.

\section{Paund-Rebka experiments}

Let us check the obtained relationships using the results of the Pound-Rebka experiment [5]. Photon passes the section $h=22.5 \mathrm{~m}$ from the source located below in time $t=h / C=7.505 * 10^{-8} \mathrm{~s}$. In this case, its speed will decrease by the value $\Delta C=t * g=7.363 * 10^{-7} \mathrm{~m} / \mathrm{s}$, the redshift factor will be obtained as $Z=\Delta C /(C-\Delta C)=2.456 * 10^{-15}$.

Let us compare it with the shift obtained in the experiment as the displacement of the spectral lines following the expression $Z=\left(v_{0}-v^{\prime}\right) / v^{\prime}=2.5 * 10^{-15}$ [5]. The factor obtained as a result of a change in the photon speed proved the same as the one obtained in the experiment. 
If the source is situated at the top of the tower and the receiver is at the bottom, the speed of the photon will increase by the value $\Delta C=t * g=7.363 * 10^{-7}$ $\mathrm{m} / \mathrm{s}$, and the blueshift factor is obtained as $Z=\Delta C /(C-\Delta C)=-2.456 * 10^{-15}$.

\section{The redshift of the Sun}

The redshift of the Sun, determined experimentally, is $2.1^{*} 10^{-6}$ [9]. The calculation to check the redshift due to the gravity of the Sun was performed numerically using an algorithm similar to the previous one (used to explain the Pound-Rebka experiments), i.e., we determine a decrease in the speed of a ray of light under the influence of the Sun's gravity, which will be seen from the Earth as a slowdown in the speed of light.

Using this algorithm, we find the time it takes for the light to cover the current calculated section and then find an increment in the speed (deceleration) of the ray on this section. We calculate the total increment in the speed of light (deceleration) as the sum of the increments in all calculated sections when a distance of 149.241 million $\mathrm{km}$ from the Sun and 258.6 thousand $\mathrm{km}$ before the Earth is reached. At this point, the acceleration of free fall of the Sun and the Earth is equal. For the sake of fairness, we note that a joint calculation is necessary simultaneously for the Sun and the Earth, which, however, will give an increase in accuracy by no more than $1 \%$. From this point, the photons begin to increase their speed under the influence of the Earth's gravity. We find the increment in the speed of light in the gravitational field of the Earth using a similar algorithm.

The following results have been obtained: an increase (a decrease) in the speed of the light in the gravitational field of the Sun is $624.27 \mathrm{~m} / \mathrm{s}$. An increase (a decrease) in the speed of the light in the gravitational field of the Earth is 0.203 $\mathrm{m} / \mathrm{s}$, and the total increase in the speed is $624.067 \mathrm{~m} / \mathrm{s}$. The redshift of the Sun will be calculated using the expression $Z=\Delta C /(C-\Delta C)=624 /(299792458-624)=2.082 * 10^{-6}$. Let us compare it with the shift obtained in the experiment as a shift of the spectral lines according to the formula $Z=\left(v_{0}-v^{\prime}\right) / v^{\prime}=2.1 * 10^{-6}$. 
Thus, the redshift of the solar radiation spectrum is also explained provided that the light emitted by the Sun is first decelerated by the Sun's gravity, and then slightly increases its speed under the influence of the Earth's gravity, while the total shift appears to be red.

The speed of light from the Sun can be determined experimentally. To this end, it is necessary to direct a ray from the Sun and a ray from an Earth source through the shutter to a device that records the difference in the arrival times of the rays. If we place the recording device, for example, at a distance of $200 \mathrm{~m}$ from the shutter along the path of the rays, we will observe the difference in the arrival time of the rays equal to 1.4 picoseconds $\left(1.4 * 10^{-12} \mathrm{~s}\right)$.

\section{Anomalous deceleration of spacecrafts}

The anomalous acceleration of the spacecrafts Pioneer-10, Pioneer-11, and others moving away almost radially from the solar system, is also explained provided that the radio beam gains additional speed under the influence of the Sun's gravity.

It is worth noting that for remote spacecrafts, we obtain a situation completely identical to that reproduced in the Pound-Rebka experiments. Therefore, we use an algorithm similar to the one presented above.

In [10], the authors state, "...Radiometric tracking data from Pioneer 10 and 11 spacecraft have consistently indicated the presence of a small anomalous Doppler frequency drift uniformly changing with a rate of $\sim 6^{*} 10^{-9} \mathrm{~Hz} / \mathrm{s} . .$.

For the computational experiment, we divide the entire path of the radio wave from the spacecraft (from the last communication session (80 a.u.)) to the Earth (the receiver is placed here) into a certain number of sections. We find the time it takes for the radio wave to pass the given section; find the magnitude of the freefall acceleration of the Sun at each section using the well-known formula; find an increment in the speed of the radio wave due to the gravity of the Sun in this 
section. We determine the total speed increment of the radio wave along the entire route from the spacecraft to the Earth.

Figure 1 presents a graph to illustrate the results of a change in the speed increment versus the distance (shown from 5 a.u. to 50 a.u.) from which the radio wave was emitted.

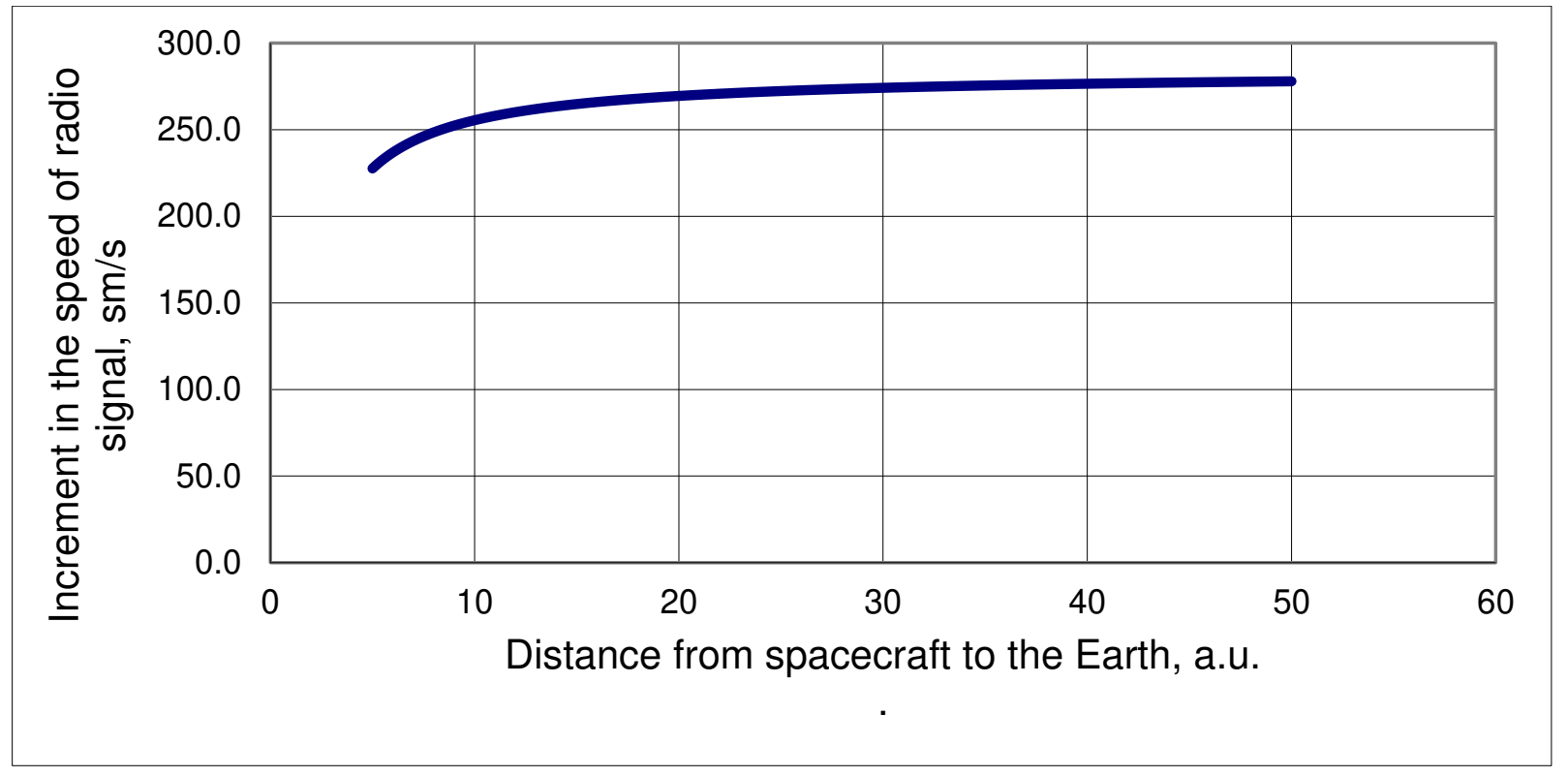

Fig. 1. A graph of a change in the speed increment depending on the distance (shown from 5 a.u. to 50 a.u.) from which the radio wave is emitted (the vertical axis is in $\mathrm{cm} / \mathrm{s}$, the horizontal axis is in a.u.).

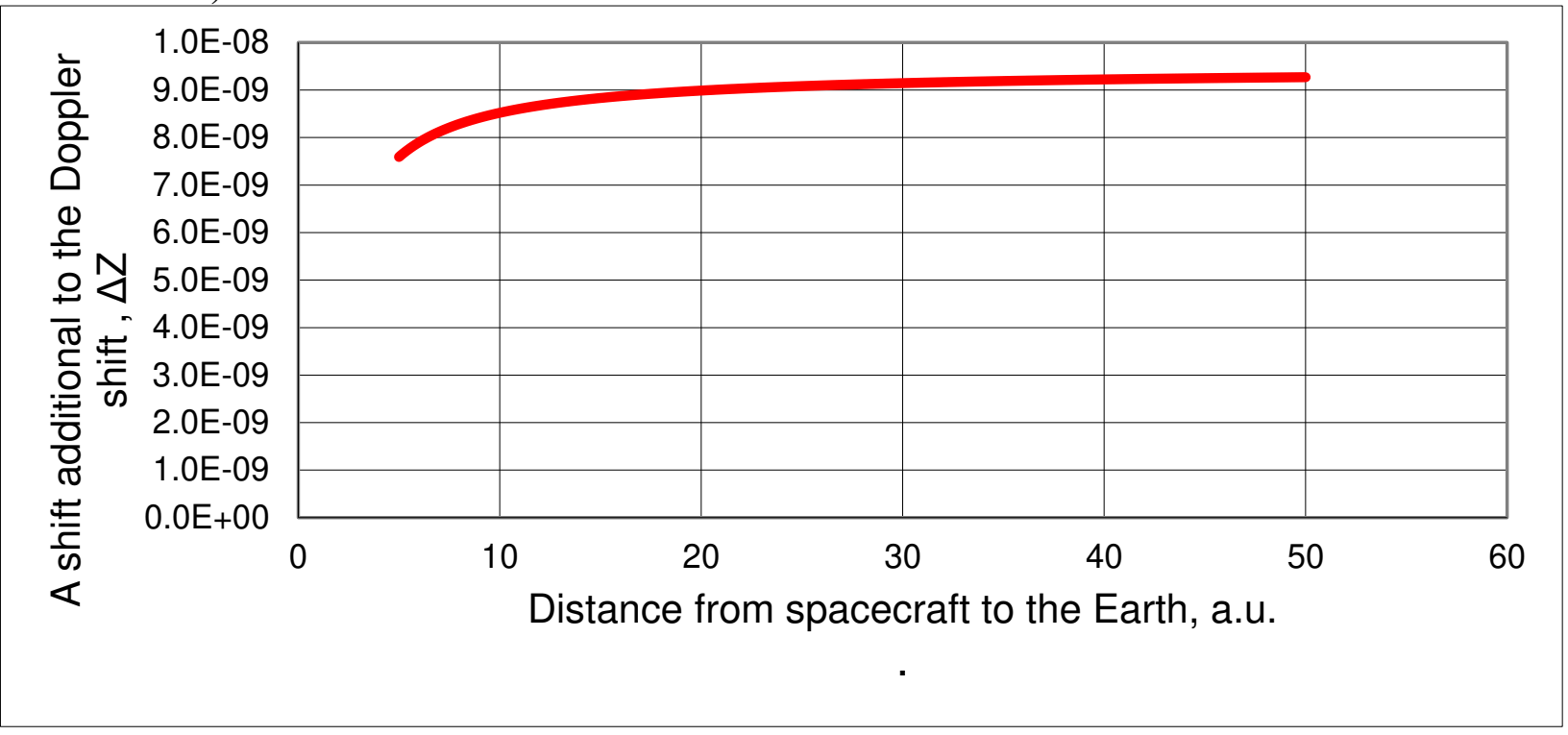

Fig. 2. A graph of a speed increment of the radio wave, which creates an additional (to the Doppler redshift caused by the movement of the spacecraft from the observer) shift (a blueshift, i.e., $\mathrm{Z}$ with the sign "-") in the spacecraft radiation received on the Earth. 
The graph shows the case where the spacecraft, the Earth, and the Sun are located in one line. In the case where the Sun is aside from this line, the increment in the speed will be lower, i.e., small variations in the parameter will be observed when the Earth moves in the orbit.

The increment in the speed of the radio wave creates an additional shift to the Doppler redshift caused by the movement of the spacecraft from the observer, i.e, a blueshift ( $Z$ with the sign "-") in the spacecraft radiation received on the Earth (Fig. 2) under the expression:

$$
Z=\left(C-C^{\prime}\right) / C^{\prime}
$$

where: $\mathrm{C}=299792458 \mathrm{~m} / \mathrm{sec}, C^{\prime}=C+\Delta V$.

For about 8 years of travel starting from the orbit of Saturn (about 10 a.u.), the spacecrafts have provided an increase in the frequency recorded by the receivers of about $1.5 \mathrm{~Hz}\left(8 * 365 * 24 * 3600 * 6 * 10^{-9}=1.514 \mathrm{~Hz}\right)$, at a frequency drift of $6 * 10^{-9}$ $\mathrm{Hz} / \mathrm{s}$.

The 'Pioneers' are known to operate at a frequency of $2.2 \mathrm{GHz}$ [11]. When the spacecrafts were in the vicinity of Saturn's orbit (10 a.u.), the frequency of the signals recorded by the receivers increased by $18.746 \mathrm{~Hz}$ by the increment in the speed of the radio wave in the gravitational field of the Sun (given the Doppler frequency decrease caused by the movement of the spacecrafts from the observer). When the spacecrafts were at a distance of 38 a.u., the frequency of the signals recorded by the receivers increased by $20.265 \mathrm{~Hz}$. The presented data were obtained from a computational experiment, however, these values are sure to be available in NASA's materials, and it will not be difficult to compare them.

Thus, in the section covering the path from the orbit of Saturn to a distance of 38 a.u., the frequency recorded at the receiver increased by $1.519 \mathrm{~Hz}$ (i.e., 20.265$18.746=1.519)$ (the one recorded by the devices was $1.514 \mathrm{~Hz}$ ).

The change in the increment of the radio signal speed over this time was $0.207 \mathrm{~m} / \mathrm{s}(2.761-2.554=0.207$, i.e., at a distance of 38 a.u. the increment in the radio wave speed is $2.761 \mathrm{~m} / \mathrm{s}$, and at the distance of 10 a.u., it is $2.554 \mathrm{~m} / \mathrm{s}$ 
(Fig.2). The change in the speed increment corresponds to the alteration in the blueshift by $6.9 * 10^{-10}$ (i.e., $0,207 / 299792458=6.9 * 10^{-10}$ ).

In this case, the change in the frequency over eight years, which will be observed at the receiver, will be $v^{\prime}=v_{0} /(1+Z)=22000000000 *\left(1 /\left(1-6.9 * 10^{-}\right.\right.$ $\left.{ }^{10}\right)=2200000001.519 \mathrm{~Hz}$.

This means that in the section from 10 a.u. to 38 a.u. the frequency recorded at the receiver increased by $1.519 \mathrm{~Hz}$ and this increase is due to a change in the increment of the radio wave speed by $0.207 \mathrm{~m} / \mathrm{s}$.

It worth noting that the frequency drift of the spacecrafts was measured sporadically, and the value $6 * 10^{-9}$ is some average value.

In actuality, the proposed method, given the Earth's orbital motion, can be used to rather accurately calculate the frequency drift for any spacecraft moving from the Sun to the solar system boundary.

Currently, some spacecrafts are far from the Sun, and it is enough to measure the blueshift in their radio signals to make sure that the research presented in the paper is correct.

Thus, the reason for the anomalous allegedly deceleration of spacecrafts moving away from the Sun has been physically explained and confirmed by the calculations, which showed good agreement of the calculation results with the measured parameters.

We can say that there is no deceleration of spacecrafts. To correctly determine the trajectory of interplanetary vehicles, it is necessary to take into account the increment in the speed of radio signals in the gravitational field of the Sun.

\section{The redshift of space objects}


In the cases considered above, the speed increment is insignificant, but it explains the effects arising. Similarly, one can explain the redshift effects of massive space objects and show that the speed of light changes.

\subsection{The redshift of a neutron star}

Let us consider a specific case using a neutron star as an example. The study in [12] shows the result of measuring the gravitational redshift of a neutron star. Measured redshift $Z=\left(\lambda^{\prime}-\lambda_{0}\right) / \lambda_{0}=0.35\left(Z=\left(v_{0}-v^{\prime}\right) / v^{\prime}=0.35\right.$ is the same for all identified lines. The radius of this neutron star was determined to be only 2.2 of the Schwarzschild radius, i.e., the ratio of the radius $\mathrm{R}(\mathrm{km})$ to the mass $\mathrm{M}$ (in solar masses) is $R / M=6.6$.

The mass of this neutron star is unknown. If it is about 1.4 solar masses, as most pulsars have, the value $R / M=6.6$ fits into the model of an ordinary neutron matter.

Thus, from this paper, we know the redshift $Z=0.35$ and the ratio of $R / M=$ 6.6. Based on these initial data, in terms of the gravity impact on the speed of light emitted by a neutron star, we will determine what mass and radius values the neutron star can have and at what speed the light of this star reaches us.

A numerical algorithm is proposed to solve this problem. It has already been applied earlier:

- Split the entire path from the surface of the star to some distance, where the increments in the speed of light will already be insignificant, into sections, for example, $100 \mathrm{~m}$ long (the solution will be more accurate if the length of the section is selected automatically, based on the set accuracy);

- Determine the time during which the light will pass the selected section. The speed of light in the first section is $299792458 \mathrm{~m} / \mathrm{s}$, the speed increment is zero;

- Find, using the well-known formula, the acceleration of gravity in this section, determine the increment in the speed in this section (increment in 
the sense of deceleration of the speed of light as it moves away from the surface of the star, which is determined by the minus sign);

- Move on to the next section, and so on through all sections. Once the final section is reached, we find the total increment (here a decrease) in the speed of light $(\Delta C)$ as a sum of the speed increments at all sections.

The results obtained are:

With a neutron star radius equal to $8.365 \mathrm{~km}$ and a mass of 1.3 solar masses, $\mathrm{km} / \mathrm{s}$ (the speed of light decreased by $77.761 \mathrm{~km} / \mathrm{s}$ ), with the redshift $\mathrm{Z}=0.35$. Since for an observer on the Earth the speed of light coming from the neutron star will be less by the magnitude of its deceleration due to the gravity of the star, the redshift is determined as $Z=\Delta C /(C-\Delta C)=77761 /(299792-77761)=0.35$. In this case, however, the ratio of $R / M=6.435$. The inequality of the obtained ratio to the value 6.6 can be explained by the insufficient calculation accuracy set.

The radii of the neutron star determined also for the masses of 1, 1.1, 1.2, 1.4 solar masses that provide the redshift $Z=0.35$ are, respectively, 6.425, 7.071, 7.718 , and $9.012 \mathrm{~km}$. At the same time, the redshift of the neutral star is in no way related to the distance to it.

\subsection{The redshift of quasars}

As for quasars, neither mass nor radius is known for them. Quasars were for the first time discovered in 1960 as radio sources coinciding in the optical range with faint stellar objects. In 1963, M. Schmidt (USA) proved that the lines in their spectra are strongly redshifted. Assuming that this redshift is caused by the Doppler effect, which arose as a result of the quasars moving away, the distance to them was determined according to Hubble law. A galaxy with a redshift of 7.085 has been recently identified [13]. 
More than 200,000 quasars have already been discovered. The closest and the brightest (3C 273) of them has a brightness of about $13^{\mathrm{m}}$ and a redshift of $\mathrm{Z}=$ 0.158 (which corresponds to a distance of about 3 billion light years). The most distant quasars, due to their supposedly gigantic luminosity, exceeding the luminosity of normal galaxies by hundreds of times, can be seen at a distance of more than 10 billion light years [13]. The studies of the nearest quasars have allowed us to find out that they are located in the nuclei of large galaxies; this is probably typical of other quasars as well. The irregular variability in the quasar brightness indicates that the region where their radiation is generated is of a small size comparable to the size of the solar system.

If we assume that the quasar is a kind of superstar that burns hydrogen it consists of, then its mass should be up to one billion solar masses [13].

Thus, the initial data for calculating the increments of speed in the gravitational field of the quasar include a mass that "will reach hundreds of millions of the Suns" and a redshift with the values varying from 0.158 to 7 . The calculation employs the algorithm used for a neutron star. In this case, we set some value for the quasar mass, for example, 10 million solar masses, and select its radius, at which we obtain a given redshift.

The following results have been obtained:

With a quasar mass equal to 10 million solar masses, we obtain its radius equal to 41.19 solar radii and $\Delta C=239921 \mathrm{~km} / \mathrm{s}$ (the speed of light decreased by $239921 \mathrm{~km} / \mathrm{s}$ ), while the redshift $\mathrm{Z}=4.0073$. Since for an observer on the Earth, the speed of the light coming from a quasar (similarly to a neutron star) will be less by the amount of its deceleration due to the gravity of the quasar, i.e., it is equal to the difference $(299792-239921)$, the redshift is determined as $Z=\Delta C /(C-\Delta C)=$ $239921 /(299792-239921)=4.0073$. In this case, the ratio $R / M=2.883$, the sizes and masses of quasars can be much smaller than it is commonly believed, and the distances to them are much shorter than those determined by $\mathrm{Z}$. 
Computational experiments, whose results are in good agreement with the experimental data, show that the redshift in the electromagnetic spectra of distant objects in the Universe is characterized by the degree of decrease in the speed of light due to the gravity of these objects, which is accompanied by a decline in the frequency recorded at the receiver.

Thus, knowing the value of the redshift $(Z)$ in the electromagnetic spectrum of a space object, one can determine the speed of light $\left(C^{\prime}\right)$ coming from this object to the observer on the Earth: $C^{\prime}=C /(1+Z)$. This formula can be applied to any values of redshift. In this case, the redshift of a quasar is by no means related to the distance to it.

\subsection{Observations by Halton Arp}

The official science understands redshift as a value characterizing the radial velocity $(V)$ of the space objects moving away in the Universe expanding after the Big Bang. In this case, the redshift is described by expression (1), and the radial velocity is determined by expression (2). The magnitude of the radial velocity is used to determine the distance to the object according to (3).

The official science does not recognize the possibility that the speed of light can be other than nominal $(299792458 \mathrm{~m} / \mathrm{s})$. However, many studies directly show its gravity dependence. These are the Pound-Rebka experiments (determination of

the Earth's gravitational redshift) and the experiments performed to measure the Sun's gravitational redshift. Moreover, we know the studies by Halton C. Arp that show the space objects (galaxies and quasars), which have different redshift factors, yet are visually located close to each other [14]. According to the standard theory of the expanding Universe, an object with a small redshift should be relatively closer to us, and an object with a large redshift should be farther away. Thus, two objects that are close to each other should have approximately the same redshift. 
In the case study by $\mathrm{H}$. Arp, the spiral galaxy NGC7603 ( $Z=0.029, V=8568$ $\mathrm{km} / \mathrm{s}, R=466$ million light years) is connected to the neighboring galaxy (object 1 , $Z=0.057 \mathrm{~V}=16601 \mathrm{~km} / \mathrm{s}, R=902 \mathrm{~m}$ light years) through a luminous bridge (Fig. 3). Judging by the difference in their redshift, the galaxies should be at significant distances from each other. The neighboring galaxy should be 436 million light years farther away (Table 1). For comparison, our Galaxy is only 2.9 million light years distant from the nearest "neighbor", the Andromeda galaxy M31 (NGC224).

Moreover, quasars were found in the luminous bridge (object $2, Z=0.243$, $V=64203 \mathrm{~km} / \mathrm{s}, R=3.5$ billion light years and object $3, Z=0.391, V=95496 \mathrm{~km} / \mathrm{s}$, $R=5.2$ billion light years).

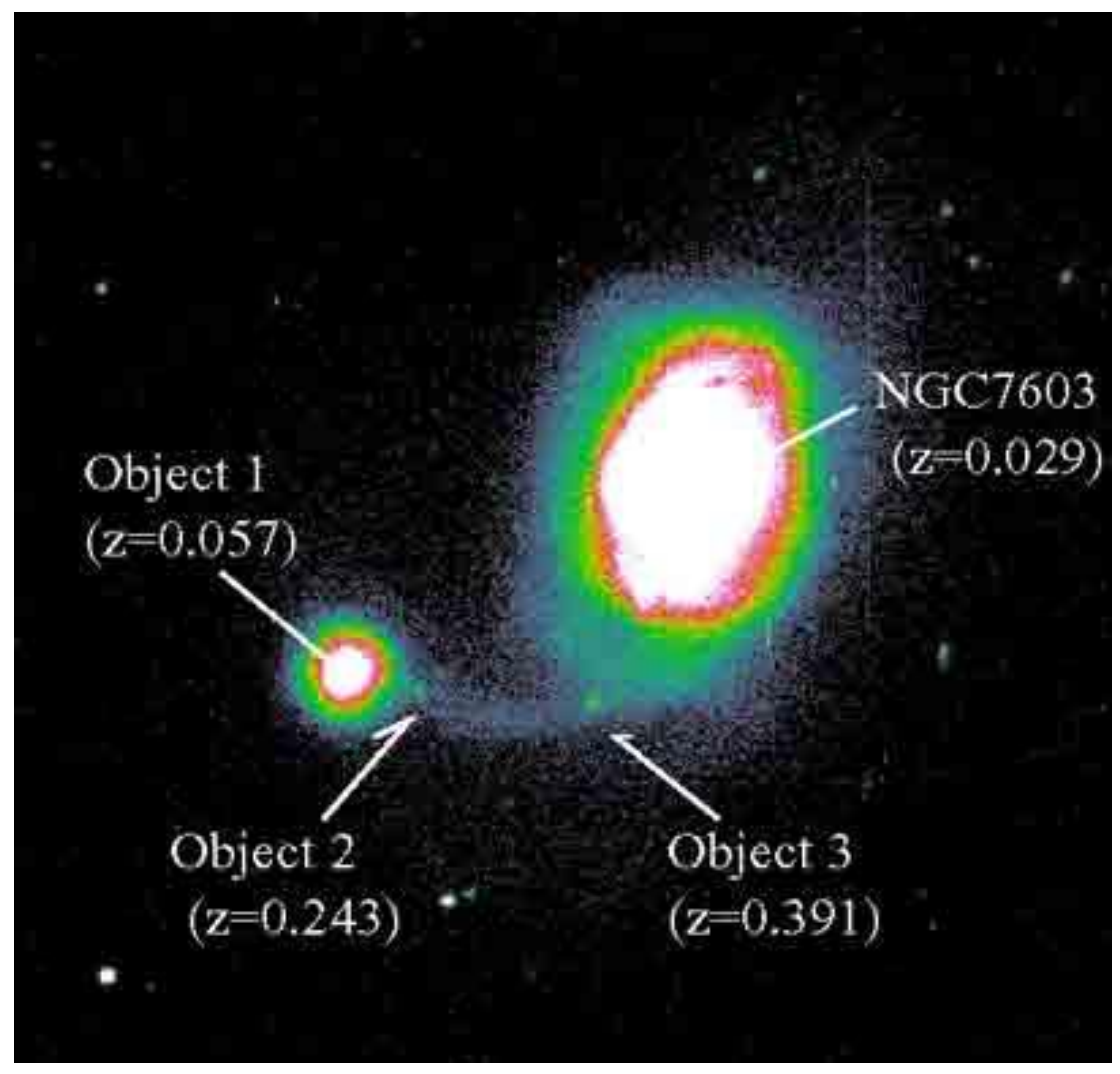

The main galaxy, NGC 7603 is an active, X-Ray bright Seyfert with a redshift of 8,000 $\mathrm{km} / \mathrm{s}$. The companion is smaller with a redshift of $16,000 \mathrm{~km} / \mathrm{s}$ and a bright rim where the filament from the Seyfert enters it. The recent measures indicate the filament is drawn out of the low redshift parent and contains the two emission line, high redshift, quasar like objects. From López-Corredoira and Gutiérrez 2002.

Fig.3. A photo from the paper by Halton Arp [14]. 
Table 1. Deceleration of the speed of light due to the gravity of space objects at various redshifts

\begin{tabular}{|c|c|c|c|c|}
\hline $\begin{array}{c}\boldsymbol{Z} \\
\text { Object } \\
\text { redshift }\end{array}$ & $\begin{array}{c}\boldsymbol{V} \\
\text { Pecular } \\
\text { speed } \\
(\mathrm{km} / \mathrm{s})\end{array}$ & $\begin{array}{c}\boldsymbol{R} \\
\text { Distance } \\
\text { to object } \\
\text { (million } \\
\text { light } \\
\text { years })\end{array}$ & $\begin{array}{c}\boldsymbol{C}^{\prime} \\
\text { Real speed of } \\
\text { light to the } \\
\text { object }(\mathrm{km} / \mathrm{s})\end{array}$ & $\begin{array}{c}\Delta C=C-C^{\prime} \\
\text { Speed } \\
\text { deceleration } \\
(\mathrm{km} / \mathrm{s})\end{array}$ \\
\hline 0.029 & 8567.9 & 465.5 & 291343.5 & 8448.9 \\
\hline 0.057 & 16601.9 & 902.0 & 283625.8 & 16166.7 \\
\hline 0.243 & 64203.7 & 3488.4 & 241184.6 & 58607.9 \\
\hline 0.391 & 95496.3 & 5188.6 & 215523.1 & 84269.5 \\
\hline
\end{tabular}

The explanation of this paradox, proposed by H. Arp, looks unconvincing and is considered controversial in official science. Arp uses Narlikar's idea of the redshift cause. He writes [15], "... One can observe large differences in the redshift from extragalactic objects located at the same distance. An internal redshift is required for an explanation. What will happen if the fundamental particles have a small mass? Simply, the transitions of low-mass electrons between orbits in the atom will result in the absorption or emission of low-energy photons, i.e., they will seem to have redshift, compared to photons emitted by heavier particles ....". We can read on the website http://haltonarp.com/: "Quasars are born with a high redshift and evolve into galaxies with a lower redshift ..." H. Arp's website contains a large number of examples of similar visually related objects but with different redshifts.

In the context of the hypothesis proposed in this paper about the relationship between the speed of light and gravity, the solution to this paradox is quite simple. All visually related objects are close to each other. and the values of redshift in the electromagnetic spectrum of these objects show the deceleration of the speed of light due to the gravity of these objects. 
For the given case of two galaxies and two quasars (Fig. 3), we obtain the values of the speed of light at an observer on the Earth (Table 1) determined by the formula $C^{\prime}=C /(1+Z)$.

Thus, with the correct use of redshift, we obtain a real speed of light $\left(C^{\prime}\right)$ from each of the objects.

\section{Rationale of new experiments}

The question naturally arises, if the speed of light from space objects is so strikingly different from the nominal, then why has not it been discovered so far? The answer can be very simple - scientists of the official science were so convinced that the speed of light is constant that they did not even ask a question in such a setting. To some extent, this was facilitated by the fact that it is impossible to measure the speed of light from an extraterrestrial object by a direct method (using optical instruments). As soon as a photon hits the lens, it further moves along the lens material at a rated speed as a result of the reemission of photons. Therefore, the methods of direct assessment are needed.

One of the possible methods can be an experiment involving the observation of an eclipse of an object with a redshift (the higher the shift, the more noticeable the effect) obscured by any planet of the Solar system, with the object observed as if crawling onto the planet's disk, i.e., the object will be seen against the background of the planet's disk edge. We use the time of this observation and the known distance to the planet to determine the speed of light coming from the object. This speed must coincide with the speed of light, determined by the expression $C^{\prime}=C /(1+Z)$.

The very first experiments to measure the speed of light from such objects as a quasar or a galaxy moving away based on the proposed method will show its significant difference from the rated speed of light. 
Thus, the gravitational redshift of spectral lines is characteristic of all gravitating objects. The larger the gravitating object mass and the smaller its dimensions, the greater shift is recorded at the receiver, i.e., the redshift is just a characteristic of the GO parameters and in no way a characteristic of the speed, at which these objects move away from the observer (except for the cases where the objects orbit, when the Doppler effect manifests itself, one can observe here both an insignificant redshift and an insignificant blueshift, i.e., the gravitational redshift can be either strengthened or weakened by the cosmological one). Moreover, there are no methods that would make it possible to distinguish the gravitational redshift from the cosmological one.

\section{A scale of gravitational redshifts}

The relationship between the speed of light from space objects and the redshift in their spectra was revealed above: $C^{\prime}=C /(1+Z)$. It shows how much the speed C $(299792458 \mathrm{~m} / \mathrm{s})$ of photons emitted by the object decreases due to its gravity. Using the developed method to calculate the speed of light propagating in a gravitational field, the ratios of the size of an object to its mass are obtained for a neutron star and a quasar.

Next, the problem of determining the entire scale of gravitational redshift for the objects of any mass and any size was solved through the calculation disregarding whether or not the objects with such parameters are found.

Solving the problem revealed that for the objects of ANY mass, the redshift will be the same at the same ratio of $R / M$, where $R$ is the radius of the object in $\mathrm{km}$, and $\mathrm{M}$ is its mass in solar masses.

Then, an array of points $Z=f(R / M)$ was obtained by setting the value of the $R / M$ ratio for one of the mass values (Table 2). The calculations were made in Excel. To obtain one point, a 7x16000 table was compiled. The values from Table 2 were used to build a graph of the relationship $Z=f(R / M)$ (Fig.4).

Table 2. Relationships between the redshift of a space object and the ratio of the object size to its mass ( $\mathrm{R}$ is the radius of the object in $\mathrm{km}, \mathrm{M}$ is the mass of the object in solar masses). 


\begin{tabular}{|c|c|}
\hline $\mathbf{R} / \mathbf{M}$ & $\mathbf{Z}$ \\
\hline 2.74 & 26.156 \\
\hline 2.75 & 12.797 \\
\hline 2.76 & 9.469 \\
\hline 2.77 & 7.782 \\
\hline 2.78 & 6.719 \\
\hline 2.79 & 5.972 \\
\hline 2.8 & 5.411 \\
\hline 2.81 & 4.969 \\
\hline 2.82 & 4.61 \\
\hline 2.83 & 4.311 \\
\hline 2.84 & 4.056 \\
\hline
\end{tabular}

\begin{tabular}{|c|c|}
\hline $\mathbf{R} / \mathbf{M}$ & $\mathbf{Z}$ \\
\hline 2.85 & 3.837 \\
\hline 2.86 & 3.645 \\
\hline 2.87 & 3.475 \\
\hline 2.88 & 3.324 \\
\hline 2.89 & 3.188 \\
\hline 2.9 & 3.065 \\
\hline 3 & 2.257 \\
\hline 3.2 & 1.536 \\
\hline 3.4 & 1.185 \\
\hline 3.6 & 0.972 \\
\hline 3.8 & 0.826 \\
\hline
\end{tabular}

\begin{tabular}{|c|c|}
\hline $\mathbf{R} / \mathbf{M}$ & $\mathbf{Z}$ \\
\hline 4 & 0.719 \\
\hline 4.5 & 0.544 \\
\hline 5 & 0.437 \\
\hline 6 & 0.312 \\
\hline 7 & 0.241 \\
\hline 8 & 0.195 \\
\hline 9 & 0.163 \\
\hline 10 & 0.139 \\
\hline 15 & 0.077 \\
\hline 20 & 0.051 \\
\hline 30 & 0.028 \\
\hline
\end{tabular}

Now, knowing the redshift of a space object, according to the graph, one can immediately determine the ratio of the object's size to its mass. Astronomers tend to find it easy to calculate the mass of an object and then, using the relationship $Z=f(R / M)$, determine its size. In this case, the size of an object is understood as the radius of its radiating surface.

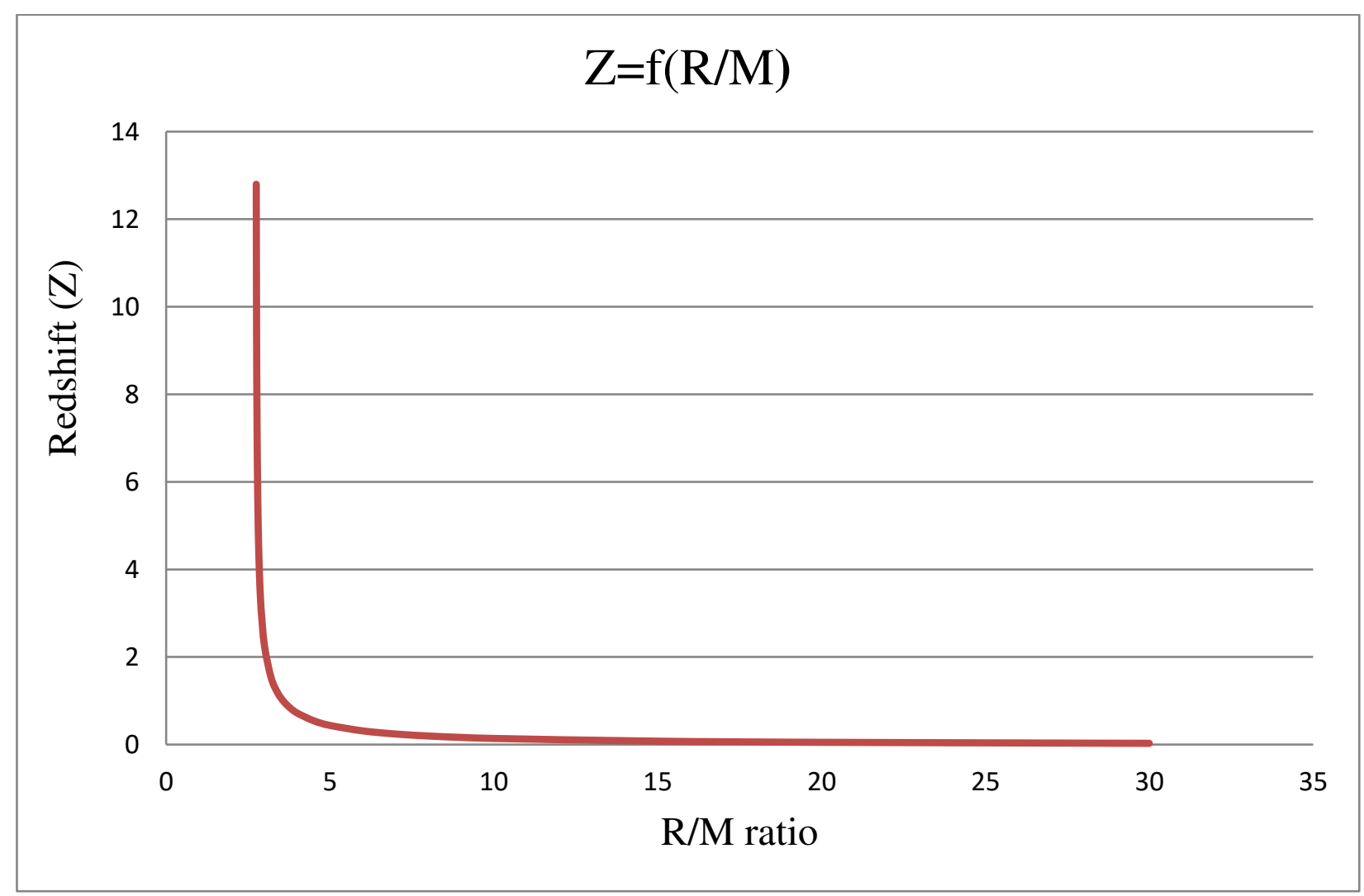


Fig.4. Graph of the relationship between the redshift of a space object and the ratio of the object's size to its mass ( $R$ is the radius of the object in $\mathrm{km}, \mathrm{M}$ is the mass of the object in solar masses).

Thus, the given relationship for the objects of any mass, which is only possible in the Universe, covers all possible variants of the redshift values due to the objects' gravity. In this case, we should take into account the shifts (red and blue) caused by the movement of the objects (from us and to us). According to the calculated forecasts, the objects with a redshift equal to $Z=26$ or more will soon be discovered (the light emitted from this object will have a speed of $11111 \mathrm{~km} / \mathrm{sec}$ or less), and they will most likely be located in our Galaxy. The highest value of redshift $Z=197.496$ was obtained by calculation for $R / M=2.7366$. The obtained relationship was also verified for such objects as the Sun and the Earth, and an exact coincidence was obtained. Therefore, now it will be incorrect to consider the redshift as a phenomenon caused by the expansion of the Universe because all possible redshifts are explained by gravity, i.e., the relationship between sizes and masses of objects and the speeds of movement of the objects in orbits (galaxies are also likely to move in their orbits around some center of the Universe).

\section{Conclusions}

The erroneous use of the Doppler effect to interpret the redshift in the electromagnetic spectra of space objects as a characteristic of their motion from the observer led to the incorrect general theory of relativity; the theory of the expanding Universe, the theory of the Big Bang, the theory of dark matter, and to the theory of an acceleratingly expanding Universe.

The correct use of redshift, as that of a predominantly gravitational nature, solves all problems and allows seeing a real picture of the Universe and getting a clear vision of its structure and evolution.

\section{References:}

1. Hubble E P Extragalactic nebulae Astrophys.J. 64321 (1926) 
2. Slipher V M Nebulae Proc. Am. Phil. Soc. 56403 (1917)

3. Einstein A Die Grundlage der allgemeinen Relativitats theorie, Annalen der Physik 354769 (1916)

4. Big Soviet Encyclopedia. M. Sovetskaya entsiklopedia 1969-1978.

5. Pound R V, Rebka G A (Jr) Phys. Rev. Lett. 3439 (1959)

6. M.v Laue History of Physics. Academic Press, 1950. - 150 p.

7. Ostashev V.E. Doppler effect in a moving medium and a change in the direction of propagation of sound emitted by a moving source. Acoustic Journal, 1988, vol. XXXIV, no. 4, pp. 700-705.

8. Bedrin A.G., Dokuchaev V.G., Aushev A.F., Zhilin A.N. Absolute radiometric calibration of the USB4000 spectrometer, Research Institute for Complex Testing of Optoelectronic Devices and Systems, 188540, Sosnovy Bor, Leningrad Region.

9. Okun L., Selivanov K., Telegdi V. Gravitation, photons, clocks. Advances in Physical Sciences. vol.169, no.10, pp.1141-1147 (1999).

10.Turyshev S. G., Toth V. T., Kellogg L. R., Eunice L., Lau E. L., Lee K. J. The Study of the Pioneer Anomaly: New Data and Objectives for New Investigation. Available at http://arxiv.org/find/grqc/1/au:+Turyshev_S/0/1/0/all/0/1 (accessed 1.12.2020)

11.John D. Anderson, Philip A. Laing, Eunice L. Lau, Anthony S. Liu, Michael Martin Nieto, Slava G. Turyshev. Study of the anomalous acceleration of Pioneer 10 and $11 / /$ Physical Review D. 2002. vol. 65, no. 8. p. 082004

12.Cottam J, Paerels F, Mendez M Gravitational redshift of the neutron star, J. Nature, 11. 2002.

13. Mortlock D. J. and others. A luminous quasar at a redshift of $\mathrm{z}=7.085$. Nature, 2011, v.474, p.616.

14.Available at: http://www.haltonarp.com/articles/research_with_Fred (accessed 1.12.2020)

15.Available at: http://www.haltonarp.com/articles/is physics changing. (accessed 1.12.2020). 
Figures

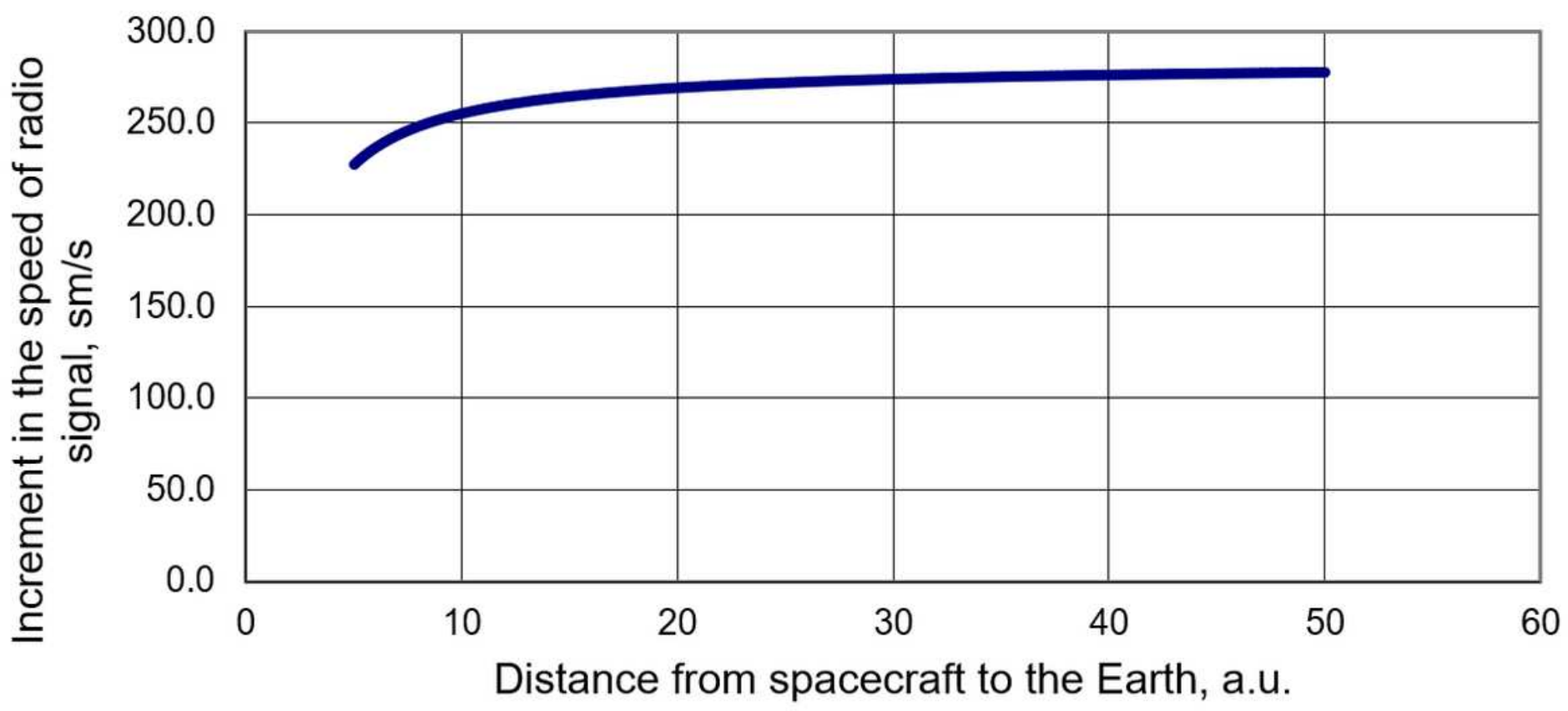

Figure 1

A graph of a change in the speed increment depending on the distance (shown from 5 a.u. to 50 a.u.) from which the radio wave is emitted (the vertical axis is in $\mathrm{cm} / \mathrm{s}$, the horizontal axis is in a.u.).

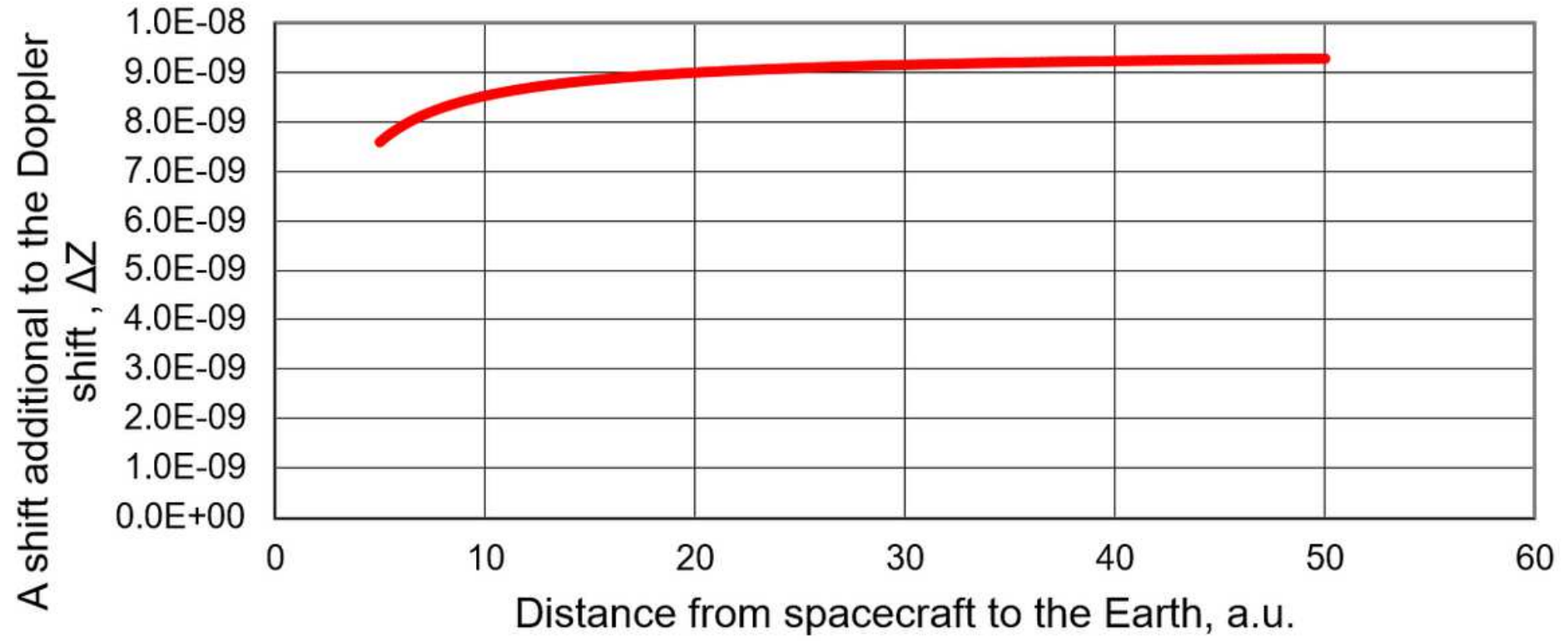

Figure 2 
A graph of a speed increment of the radio wave, which creates an additional (to the Doppler redshift caused by the movement of the spacecraft from the observer) shift (a blueshift, i.e., $Z$ with the sign "-") in the spacecraft radiation received on the Earth.

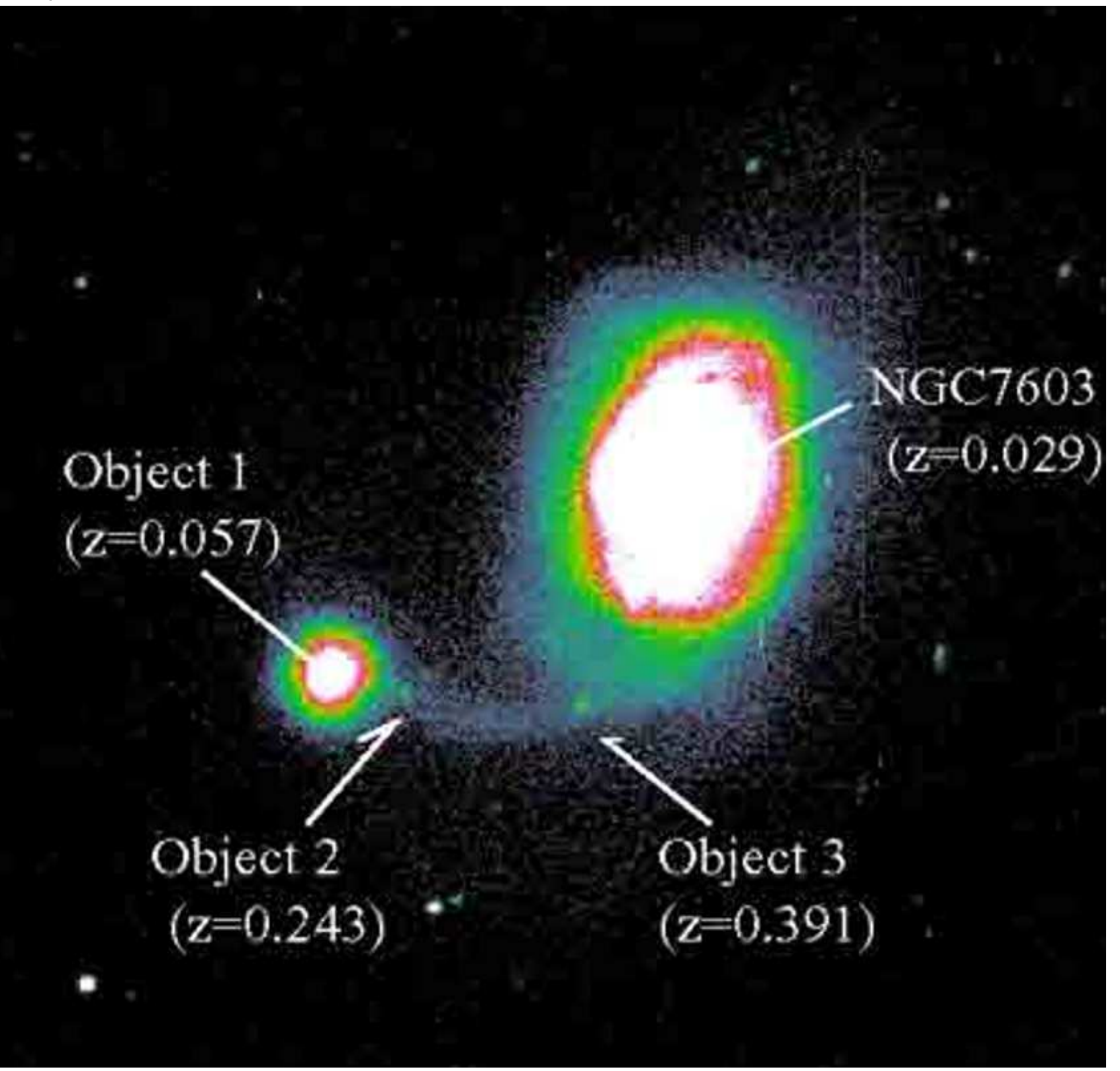

\section{Figure 3}

A photo from the paper by Halton Arp [14]. The main galaxy, NGC 7603 is an active, X-Ray bright Seyfert with a redshift of $8,000 \mathrm{~km} / \mathrm{s}$. The companion is smaller with a redshift of $16,000 \mathrm{~km} / \mathrm{s}$ and a bright rim where the filament from the Seyfert enters it. The recent measures indicate the filament is drawn out of 
the low redshift parent and contains the two emission line, high redshift, quasar like objects. From LópezCorredoira and Gutiérrez 2002.

\section{$\mathrm{Z}=\mathrm{f}(\mathrm{R} / \mathrm{M})$}

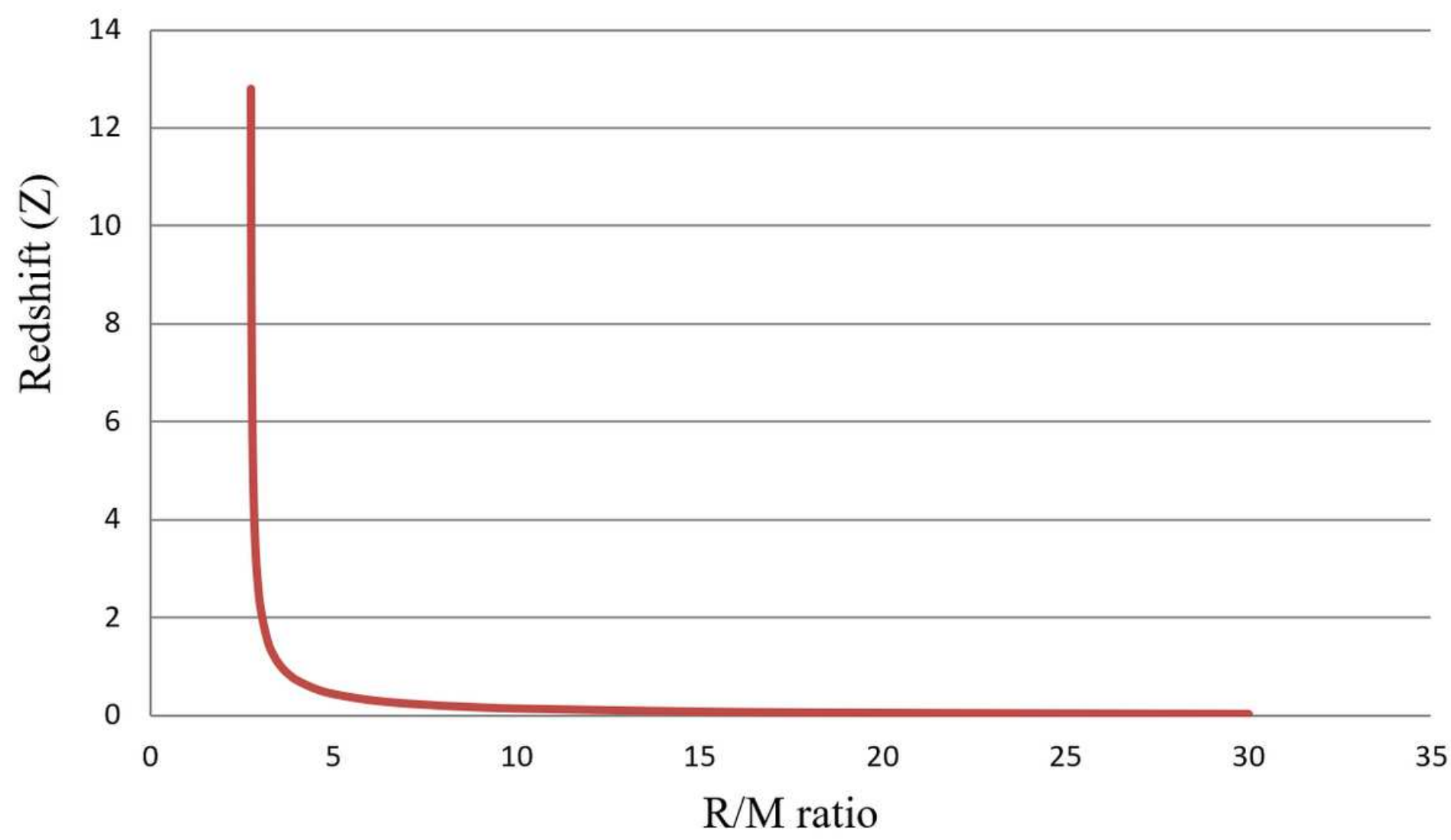

\section{Figure 4}

Graph of the relationship between the redshift of a space object and the ratio of the object's size to its mass ( $R$ is the radius of the object in $\mathrm{km}, \mathrm{M}$ is the mass of the object in solar masses). 